EDITORIAL

\title{
Pregnancy in women with congenital heart disease: the importance of evaluation and counselling
}

\author{
K Stout
}

Issues surrounding the growing population of pregnant women with congenital heart disease are becoming increasingly important as more women with surgically corrected heart conditions reach childbearing age

Heart 2005;91:713-714. doi: 10.1136/hrt.2004.047886

with pregnancy. Systemic vascular resistance decreases during pregnancy but will increase dramatically at the time of delivery. Delivery also results in a sudden increase in venous return. Determining which women are at increased risk of adverse outcomes and providing advice about pregnancy is optimally done before conception as part of a full cardiac evaluation.

In addition to the risks of pregnancy itself, there is an increased incidence of congenital heart disease in children of women with a congenital abnormality ranging from approximately $3 \%$ overall to $50 \%$ in women with single gene defects with autosomal dominant inheritance, such as Marfan syndrome.

Congenital heart disease presents not only a wide spectrum of lesions and repairs, there also is a wide range of pathophysiology even in patients with the same "condition". Unfortunately, there are relatively little data to guide recommendations regarding pregnancy risk for a specific patient. Firm recommendations based upon solely the underlying anatomy are rarely possible as the risk to mother and fetus varies depending on each individual's underlying physiology. Patients with simple lesions without substantial haemodynamic consequence, such as small atrial septal defects, are at low risk of cardiac deterioration or substantial fetal difficulties. At the other end of the spectrum are patients who are at substantial risk of significant maternal cardiac complications, including death, such as those with Eisenmenger's syndrome. Women with conditions such as repaired tetralogy of Fallot may be low, medium, or high risk depending on the underlying physiology, ventricular function, and valvar function. Cyanotic patients are at increased risk of fetal complications including spontaneous abortion and intrauterine growth retardation. Each patient needs to be individually assessed for the ability to tolerate the increased cardiac output, increased heart rate, lower systemic vascular resistance, lower blood pressure, and increased blood volume that occur with pregnancy.

\section{TETRALOGY OF FALLOT}

Tetralogy of Fallot is of particular interest as it is the most common cyanotic congenital heart disease and was among the first complex defects to be successfully surgically repaired. In this issue of Heart, Meijer and colleagues ${ }^{2}$ report on the pregnancy, fertility, and recurrence risk in a series of 29 women with repaired tetralogy of Fallot. Notable in their series is the finding that, overall, pregnancy was well tolerated in this diverse group of women. However, severe 
pulmonary insufficiency was a risk factor for decompensation during pregnancy. These findings emphasise the need to assess women with congenital heart disease, even after a "successful" repair, on a regular basis to ensure that any cardiac lesions that may limit cardiac reserve sufficiently to complicate pregnancy can be corrected before conception. As there are unlikely to be large randomised controlled trials to guide our care of these patients, detailed case series such as reported here will further our ability to make recommendations to our patients.

\section{A PRACTICAL APPROACH TO RISK ASSESSMENT}

At this point, there are some simple steps that provide initial risk stratification in women with congenital heart disease, with the history and physical examination being the starting point. A patient's functional capacity, exercise tolerance, New York Heart Association classification, degree of cyanosis, medication needs, and history of arrhythmias should all be taken into account. Not surprisingly, patients who are active and unlimited in their daily lives, on minimal cardiac medications, without arrhythmias or other cardiac symptoms are at a lower risk than those who require significant cardiac medications to maintain more limited functional capacity.

The second step is to consider the underlying anatomy and physiology of the disease process, typically with echocardiographic evaluation. Magnetic resonance imaging, computed tomographic scanning, or exercise testing, and ambulatory electrocardiographic monitoring may all play a role as well. Certain cardiac abnormalities are low risk whereas others pose such a high risk that pregnancy is ill advised.

Lesions with minimal haemodynamic effect such as small ventricular septal defects, atrial septal defects, and bicuspid aortic valves without stenosis, insufficiency, or aortic enlargement pose little risk. These patients have near normal physiology and therefore the risk of pregnancy is only minimally increased. These patients can receive routine obstetric care, including endocarditis prophylaxisas indicated.

The moderate risk category includes patients with repaired tetralogy of Fallot without significant pulmonary insufficiency or stenosis, ${ }^{3}$ complex congenital heart disease with the anatomic right ventricle serving as systemic ventricle, and mild left sided valve stenosis. In these patients pregnancy may be well tolerated, although as suggested in the article by Meijer and colleagues, ${ }^{2}$ repair of significant residual lesions should be considered before pregnancy. Patients with moderate risk conditions may be on cardiac medications that pose management difficulties. Other medications such as angiotensin converting enzyme inhibitors are contraindicated during pregnancy, and alternative medications are frequently necessary. Women who are chronically anticoagulated pose unique challenges in effectively balancing the thrombotic risks of the underlying cardiac condition and pregnancy, and the risks of coumadin during pregnancy. ${ }^{45}$ Experience in the use of cardiac medications in pregnancy and close collaboration between the cardiac and obstetric teams is necessary to ensure a good outcome for both mother and baby. Women at moderate risk should be monitored at centres with experience in managing pregnancy in women with congenital heart disease.

There is a significant risk of maternal cardiac decompensation and death during pregnancy and in the first month post partum in women with Eisenmenger's syndrome, ${ }^{6}$ severe pulmonary hypertension, severe aortic stenosis ${ }^{7}$ or left ventricular outflow tract obstruction, Marfan's syndrome with aortic dilation greater than $4 \mathrm{~cm}$, or symptomatic systemic ventricular dysfunction with an ejection fraction $<40 \%{ }^{8-10}$ These patients should be counselled against pregnancy. If high risk patients become pregnant, they require expert care at experienced high risk obstetric centres with close collaboration between cardiac and obstetric teams.

\section{FUTURE DIRECTIONS}

The issues surrounding this growing population of patients will only increase in importance as more women with surgically corrected congenital heart disease reach childbearing age. The issues surrounding pregnancy in these patients cannot be answered by prospective randomised trials, given the variability in anatomy and physiology in women with congenital heart disease and the inappropriateness of randomisation in this setting. Thus, we must continue to increase our knowledge base through carefully reported case series and clinical registries with long term outcomes data.

\section{REFERENCES}

1 The Task Force on the Management of Grown Up Congenital Heart Disease of the European Society of Cardiology. Management of grown up congenital heart disease Eur Heart J 2003;24:1035-84.

2 Meijer JM, Pieper PG, Drenthen W, et al. Pregnancy, fertility, and recurrence risk in corrected tetralogy of Fallot. Heart 2005;91:801-5.

3 Veldtman GR, Connolly HM, Grogan $M$, et al. Outcomes of pregnancy in women with tetralogy of Fallot. J Am Coll Cardiol 2004;44:174-80.

4 Cotrufo $M$, de Feo $M$, de Santo LS, et al. Risk of warfarin during pregnancy with mechanical valve prostheses. Obstet Gynecol 2002;99:35-40.

5 Ginsberg JS, Chan WS, Bates SM, et al. Anticoagulation of pregnant women with mechanical heart valves. Arch Intern Med 2003;163:694-8.

6 Yentis SM, Steer PJ, Plaat F. Eisenmenger's syndrome in pregnancy: Maternal and fetal mortality in the 1990s. Br J Obstet Gynaecol 1998;105:921.

7 Easterling TR, Chadwick HS, Otto CM, et al. Aortic stenosis in pregnancy. Obstet Gynecol 1988;72:113.

8 Presbitero P, Somerville J, Stone S, et al. Pregnancy in cyanotic congenital heart disease. Outcome of mother and fetus. Circulation 1994;89:2673.

9 Siu SC, Sermer M, Harrison DA, et al. Risk and predictors for pregnancyrelated complications in women with heart disease. Circulation 1997;96:2789-94.

10 Siu SC, Sermer M, Colman JM, et al. Prospective multicenter study of pregnancy outcomes in women with heart disease. Circulation $2001 ; 104: 515-21$. 